\title{
State of the first aberration-corrected, monochromized 200kV FEG-TEM
}

\author{
Gerd Benner, Alexander Orchowski ${ }^{*}$, Max Haider, Peter Hartel ${ }^{\#}$ \\ * LEO Electron Microscopy Group, Carl Zeiss S-M-T AG, D-73446 Oberkochen, Germany \\ ${ }^{\#}$ CEOS GmbH, D-69126 Heidelberg, Germany
}

We present first results from the qualification of a new $200 \mathrm{kV}$ FEG-TEM instrument equipped with a monochromator for illumination and a Cs-Corrector for the imaging system. The SATEM (Sub-

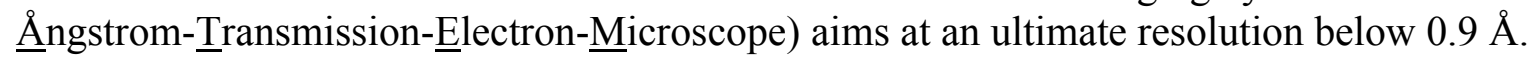

Recently it has been shown that hardware correction of the spherical aberration of the objective lens of a transmission electron microscope can be successfully implemented [1]. Following a design of Rose [2], the corrector module incorporates two magnetic hexapoles and several additional round lenses. The hexapoles induce a residual rotationally symmetric negative spherical aberration, while the transfer lenses are used to tune and match the specific intermediate optical planes of the ray path. By appropriate excitation of the hexapoles, the spherical aberration of the overall system formed by objective lens and corrector optics can be continously adjusted to a numerical value of the according coefficient $\mathrm{C}_{\mathrm{s}}$ down to zero and even to negative values. The Phase Contrast Transfer Function (PCTF) thus can be tuned via $\mathrm{C}_{\mathrm{s}}$ up to the fundamental limit given by incoherent damping. Figure 1 shows the PCTF for the uncorrected case (a) and for nearly vanishing $C_{s}(b)$ in Scherzer Focus. Further improvement is achieved by reducing the damping at higher spatial frequencies due to chromatic aberrations through use of a Monochromator. By narrowing the FWHM of the energy spread from $\Delta \mathrm{E}=0.8 \mathrm{eV}$ (inherent $\mathrm{FEG}$ value) down to $0.3 \mathrm{eV}$, the envelope function Ec of temporal coherence reaches much further. Therefore a point resolution below $1 \AA$ (Fig 1c) is the ultimate goal of this project.

The SATEM instrument (Figure 2) is based on a newly developed highly stable $300 \mathrm{~mm}$ column, equipped with a condensor system for Köhler illumination, an high resolution objective lens and a rotation-free projective system with an incorporated in-column energy filter of the in second-order corrected $\Omega$-type [3]. The $200 \mathrm{kV}$ FEG Schottky-emitter gun houses an dispersion-free monochromator of electrostatic $\Omega$-type [4] and the corrector optics are integrated into the TEM below the objective lens adding an additional length of $270 \mathrm{~mm}$ to the column. Furthermore in order to obtain highest mechanical stability of the electron optical setup, a "hanging-column concept" has been realized, where the TEM-column is supported like a pendulum in a highly stable frame,which has been optimized for maximum stiffness while providing the necessary accessibility to the column.

We demonstrate performance examples of the instrument. Progress will be reported on the achievement of the ultimate capabilities with respect to the theoretical limits.

References

[1] M. Haider et al., Nature 392 (1998), 768

[2] H. Rose, Optik 34 (1990), 19

[3] G. Lang et al., Microsc. Microanal. 8 (Suppl. 2) (2002), 586CD

[4] F. Kahl \& H. Rose, Proc. EUREM Brno/Cz, Vol.III (2000), 1459

Corresponding author: Orchowski@leo.de 
Figure 1:

SATEM PCTFs in Scherzer Focus, Parameters used: illumination aperture $\Theta_{c}=0.2 \mathrm{mrad}$, coeff. of chromatic aberration $\mathrm{C}_{\mathrm{c}}=1.2 \mathrm{~mm}$

a) $\mathrm{C}_{\mathrm{s}}=1.2 \mathrm{~mm}, \mathrm{Df}=-67 \mathrm{~nm}$, $\Delta \mathrm{E}=0.8 \mathrm{eV}$

b) $\mathrm{C}_{\mathrm{s}}=0.01 \mathrm{~mm}, \mathrm{Df}=-6 \mathrm{~nm}$, $\Delta \mathrm{E}=0.8 \mathrm{eV}$

c) $\mathrm{C}_{\mathrm{s}}=0,01 \mathrm{~mm}, \mathrm{Df}=-6 \mathrm{~nm}$, $\Delta \mathrm{E}=0.3 \mathrm{eV}$

Es $=$ spatial coherence enveloppe, $\mathrm{Ec}=$ temporal coherence enveloppe

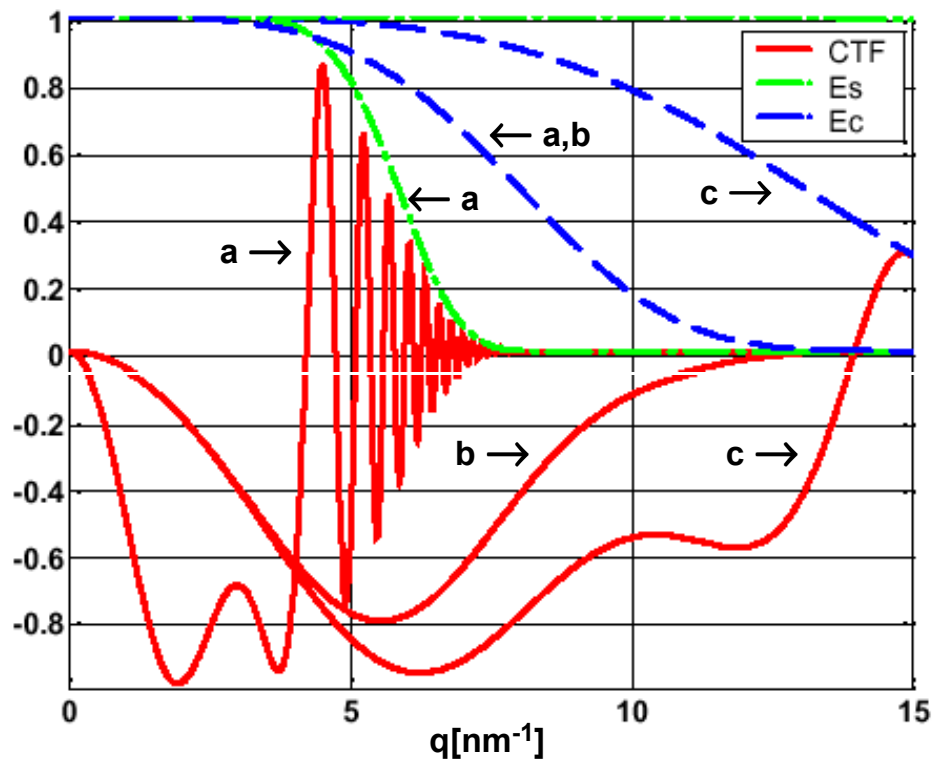

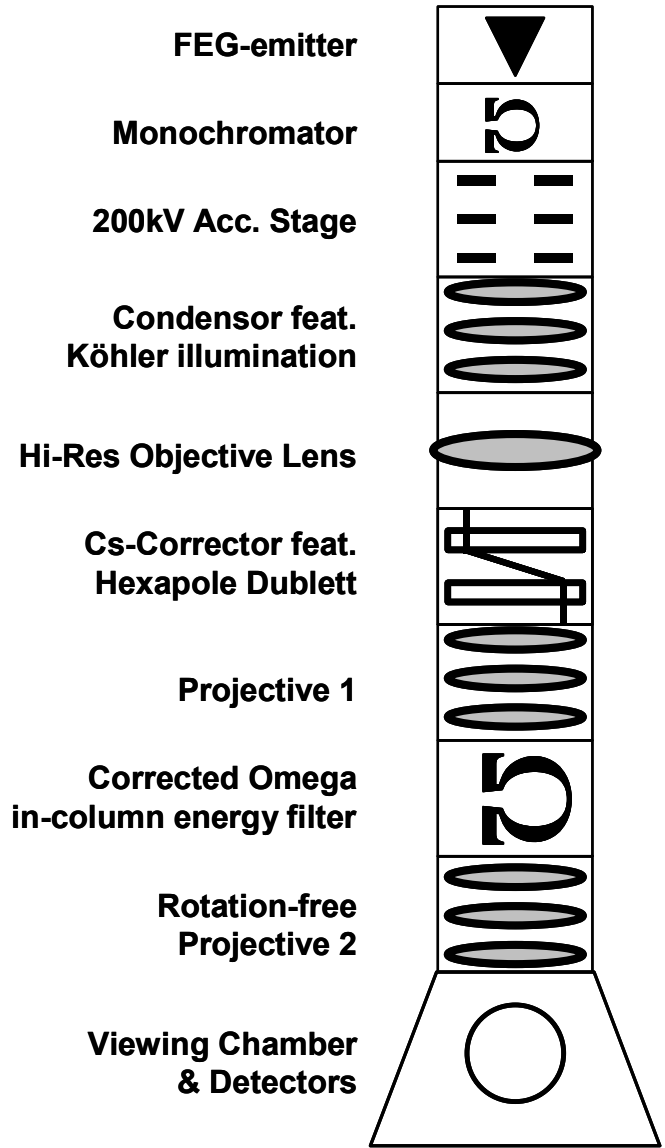

Figure 2:

Schematic setup of the modules forming the SATEM instrument. Photograph shows the column after installation in a highly stable support frame.

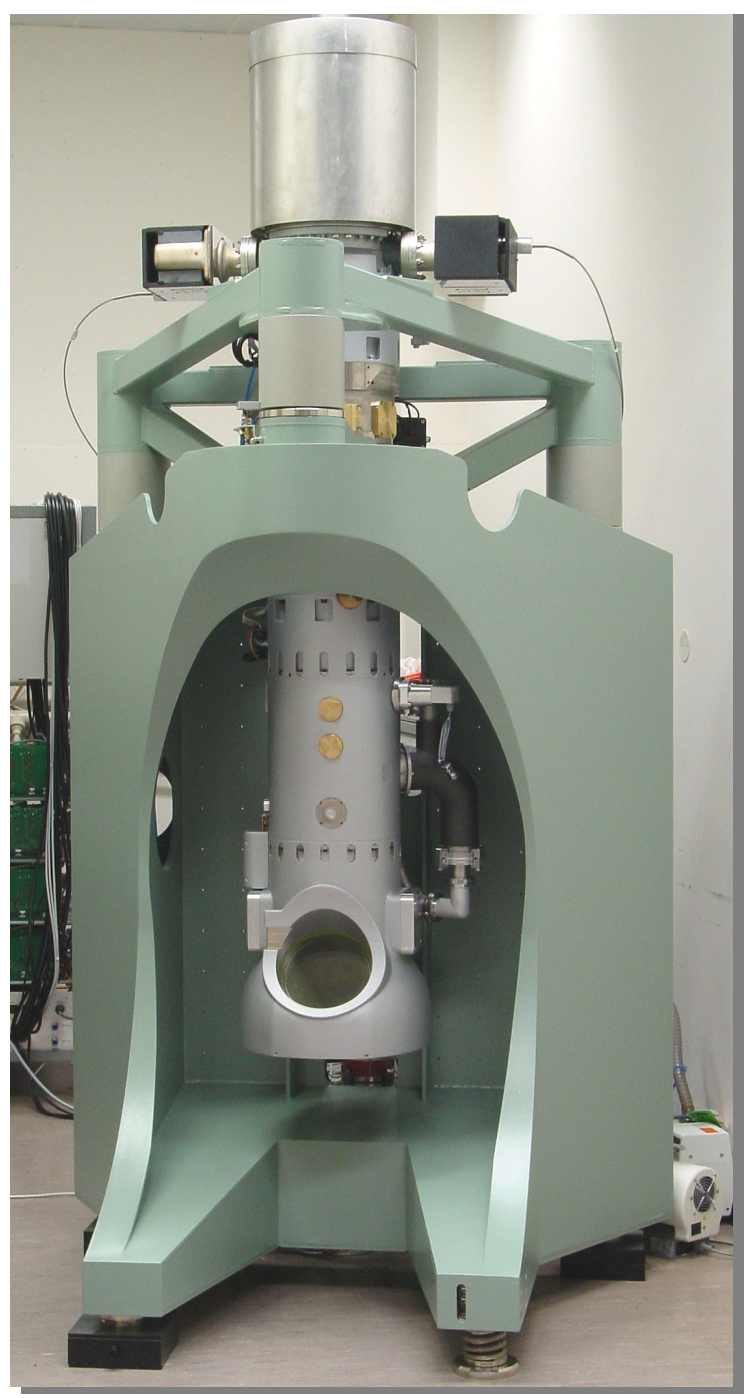

